PPPL- -2672

DE90 005807

\title{
Control of Energetic Ion Confinement by Ion Cyclotron Range of Frequency Waves
}

\author{
C.S. Chang* \\ Princeton Plasma Physics Laboratory \\ Princeton University \\ Princeton, NJ 08543
}

It is shown that ICRF waves can induce fast convective radial transport of energetic ions in a toliamak geometry without affecting the background ion transport. Spatially inhomogeneous ICRF waves with directional parallel wave vectors are needed; otherwise, a net parallel flow of the energetic ions has to be present.

\section{DISCLAIMUE}

\begin{abstract}
This report was prepared as an account of work sponsored by an agency of the United States Government. Neither the United States Government nor any agency thereof, nos any of their employees, makes any warranly, express or implied, or assumes any legal liability or respons:bility for the accuracy, completeness, or usefulness of any information, apparatus, product, or process disclosed, or represents that its use would not infringe privately owned rights. Reference herein to any specific commercial product, process, or service by trade name, trademark. manufacturer, or otherwise does not necessarily constitute or imply its endorsement. recommendation, or fayoring by the United States Government ar any agency thereof. The views and opinions of authors expressed herein do not necessarily state or feflecl those of the United States Government or any agency thereof.
\end{abstract}

- Courant Institute of Mathematical Sciences, New York University 
Active control of the energetic ion transport in a tokamak can help achieve sustained thermonuclear reaction through the heating profile control, electrostatic potential control, instability control, and alpha ash removal and burn control. In the present work. it will be demonstrated that ICRF waves can be used to control selectively the energetic ion transport. We pick ICRF waves in the present investigation because of their well-verified strong interaction with high energy ions in a magnetic confinement device.

In order to produce a net radial transport for particles with well-confined orbits in a tokamak geometry, the particles need to experience a net parallel friction force. ${ }^{1}$ ICRF waves can produce a net parallel friction in the following situations. The first case arises when the lowest order distribution of the resonance particles are not symmetric in $v_{\|}$. The second case arises when the ICRF wave power is not distributed uniformly in the plasma.

The basic physical mechanism for the first case is rather obvious, but the second case needs some introduction. When a charged particle interacts with a spatially inhomogeneuus medium, it can undergo large drift motions from one magnetic field line to another and thus induce large cross-field transport. A representative example is the large neoclassical enhancerient of plasma transport over the classical transport due to spatial inhomogeneity in the magnetic field. For similar reasons, it is likely that a spatially inhomogeneous ICRF wave power can also induce cross-field drift and, thus, cross-field transport of resonant ions. This mechanism can selectively enhance fast ion transport through the Doppler shift effect. Large Doppler broadening associated with the higher energy particles makes different signs of parallel velocity $v_{\|}$be resonant with the nonuniform waves at different locations in major radius, and thus the absorbed wave power is not symmetric in the sign of $v_{n}$. Ions with small kinetic energy have a small spatial Doppler-spread of the resunance locations; hence, they all receive essentially the same ICRF wave power. 
We use the quasilinear form of the steady-state drift-kinetic equation

$$
\left(v_{i l} \hat{n}+\vec{v}_{d}\right) \cdot \nabla f=C(f)+Q(f)+\mathcal{S},
$$

where $f$ is the quasi-static part of the high-energy ion distribution function averaged over the gyrophase, $v_{\|}$is the parallel component of particle velocity to the magnetic field $\vec{B}, C$ is the Coulomb collision operator, $Q$ is the RF-heating operator corresponding to the RF wave used for the transport control (controlling wave), and $\mathcal{S}$ represents spatially homogeneous main driving operators generating the high energy ions. $\mathcal{S}$ can be a strong ICRF-heating operator or particle source operator, representing ionization from neutral beam particles or alpha birth from fusion. $\mathcal{S}$ is defined here to be spatially homogeneous. If there is a spatially inhomogeneous part in the source function $\mathcal{S}$, we include it in $Q$ because this part can strongly enhance the radial transport.

In order to solve Eq. (1) for $f$, we assume that the banana width $\Delta_{6}$ is small compared to the radial scale length $L$ for energetic ion pressure, $\delta \equiv \Delta_{b} / L \ll 1$, and we expand $f$ in $\delta: f=f_{0}+f_{1}+f_{2}+\cdots$. The source term $S$ balances against the collision term to yield a steady-state distribution function of the energetic ions and thus formally $\mathcal{S}$ is of order $C$. However, we assume that the heating rate from the controlling RF waves is smaller than $S$ by one order in $\delta: Q \sim \delta C \sim \delta \dot{\nu}_{c} \omega_{T}$, where $\omega_{T} T$ is the trancit frequency and $\hat{\nu}_{\varepsilon}=\nu_{c} / \omega_{T}$ is the normalized collision frequency of the energetic ions. This ordering allows us to keep the lowest order distribution function undisturbed during the driven transport time scale, and justifies the neglect of the $\partial / \partial t$ term in the drift kinetic equation.

As we expand Eq.(1) in $\delta$, the two lowest order equations in $\delta$ become:

$$
v_{\|} \hat{n} \cdot \nabla f_{0}=C\left(f_{0}\right)+\mathcal{S} \cdot r_{\|} \dot{n} \cdot \nabla f_{1}-C\left(f_{1}\right)=Q\left(f_{0}\right)-\vec{v}_{d} \quad \nabla f_{0} .
$$

There are two linearly independent driving terms in the linear equation $(2): Q\left(f_{0}\right)$ and $\bar{v}_{d} \cdot \nabla f_{0}$. We write $f_{1}$ as combination of RF-driven and vertical-drift-driven terms; 
$f_{1}=f_{\mathrm{RF}}+f_{d}$, where $f_{R F}$ and $f_{d}$ satisfy

$$
\begin{aligned}
& v_{\|} \hat{n} \cdot \nabla f_{\mathrm{RF}}-C\left(f_{\mathrm{RF}}\right)=Q\left(f_{0}\right), \\
& v_{\|} \hat{n} \cdot \nabla f_{d}-C\left(f_{d}\right)=-\vec{v}_{d} \cdot \nabla f_{0} .
\end{aligned}
$$

The vertical-drift-driven distortion term $f_{\mathrm{d}}$ yields neoclassical particle transports similar to those in Refs. 2, 3, and 4. This neoclassical transport rate is too slow for an active control of the energetic ions and, thus, not of much interest. In the present work, we only consider the effect of $f_{\mathrm{RF}}$ on the energetic ion transport.

For the high energy ions, the Coulomb collision frequency is much smaller than the particle transit frequency. This observation allows us to expand $f$ in $\hat{\nu}_{e}: f=$ $f^{(0)}+f^{(1)}+\cdots$. The zeroth and first order expansion of Eq. (2) in $\hat{\nu}_{c}$ yields

$$
\begin{gathered}
v_{\|} \hat{n} \cdot \nabla f_{0}^{(0)}=0, \\
v_{\|} \hat{n} \cdot \nabla f_{0}^{(1)}=C\left(f_{0}^{(0)}\right)+\mathcal{S} .
\end{gathered}
$$

$C$ and $\mathcal{S}$ are constant along $\vec{B}$. Equation (4) shows that $f_{0}^{(0)}$ is also constant along $\vec{B}$. Equation (5) then determines $f_{0}^{(0)}$ from bounce average operation, $\{\cdots\}$,

$$
C\left(f_{0}^{(0)}\right)+\mathcal{S}=0
$$

Equation (4) also shows that $f_{0}^{(0)}$ has to be symmetric in $v_{\|}$in the banana regime. due to the bounce conditions at the banana tips. With Eq. (6), Eq. (5) is identical to Eq. (4). And, with no sources in Eq. (5), we can safely conclude $f_{0}^{(1)}=0$. Hence. we use the simplified notation $f_{0}$ for $f_{0}^{(0)}$.

Expansion of Eq. (3) in the collision frequency yields

$$
v_{p} \bar{n} \cdot \nabla f_{\mathrm{RF}}^{(0)}=0
$$

and

$$
\nu_{\|} \hat{n} \cdot \nabla f_{\mathrm{RF}}^{(1)}=C\left(f_{\mathrm{RF}}^{(0)}\right)+Q\left(f_{0}\right)
$$


As before, Eq. ( 7$)$ shows $f_{\mathrm{RF}}^{(0)}$ is constant along $\vec{B}$ and symmetric in $u_{\|}$in the trapped regime. This function $f_{R F}^{(0)}$ represents the lowest order distortion of the particle distribution function by the controlling $R F$ wave, and $f_{R F}^{(0)}$ is determined by the hounce average of $\mathrm{Eq}$. (8): $\left\{C\left(f_{\mathrm{RF}}^{(0)}\right)+Q\left(f_{0}\right)\right\}=0$. The bounce average operation defined here is taken between a complete poloidal transit cycle for the passing particles, and over a complete banana orbit for the trapped particles. Since $C, f_{\mathrm{RF}}^{(0)}$, and $f_{0}$ are constant along $\vec{B}$, the above equation for $f_{\mathrm{RF}}^{(0)}$ is reduced to

$$
C\left(f_{\mathrm{RF}}^{(\mathbf{0})}\right)+\{Q\}\left(f_{\mathrm{D}}\right)=0 .
$$

The particle flux driven by RF heating may be calculated from the relation

$$
\Gamma_{v}=\vec{\Gamma} \cdot \nabla \psi=\left\langle\int d^{\mathrm{a}} v \vec{v}_{d} \cdot \nabla \psi f_{\mathrm{RF}}^{(1)}\right\rangle
$$

where $\langle\cdots\rangle$ denotes flux surface average. Using $\vec{v}_{d} \cdot \nabla \psi=I_{v_{\|}} \hat{n} \cdot \nabla\left(v_{\|} / \Omega\right)$ with $I=R B_{T}$ and integrating by parts, we obtain

$$
\begin{aligned}
\Gamma_{\psi} & =-\left\langle\int d^{3} v \frac{v_{\|} I}{\Omega} v_{\|} \tilde{n} \cdot \nabla f_{\mathrm{RF}}^{(1)}\right\rangle=-\left\langle\int d^{3} v \frac{v_{\| I} I}{\Omega}\left[Q\left(f_{0}\right)+C\left(f_{\mathrm{RF}}^{(0)}\right)\right]\right\rangle \\
& =-\left\langle\int d^{3} v \frac{v_{\|} I}{\Omega}\left[Q\left(f_{0}\right)-\{Q\}\left(f_{0}\right)\right]\right\rangle
\end{aligned}
$$

where Eq. (8) and Eq. (9) have been used for the second and third equality relations. respectively. The direct collisional effect on $f_{0}$, even if it may be asymmetric in $v_{\|}$, does not produce a net radial transport because of the relation, from Eq. (6). $\int d^{3} v\left(v_{\|} I / \Omega\right)\left[C\left(f_{0}\right)+\mathcal{S}\right]=0$. The first term in Eq. (10) represents the direct friction effect on $f_{0}$ from scattering by controlling RF waves, and has a similar physical origin to the mechanism of Ref. 5 . The appearance of the second term may provole deceiving physical interpretation, but it is really the collisional friction effect on the controlling RF-wave-disturbed $f_{R F}^{(0)}$.

In most of the ICRF-heating experiment, the parallel wave number is small so that $\left|k_{\|} v_{\|}\right| \ll \ell \Omega$ holds, where $\ell$ is the cyclotron harmonic number. In this case, 
we can neglect the direct change of $v_{\|}$caused by RF heating, and thus $v_{\|}$and $Q$ commute. Since the operator $Q$ conserves particle number, the second term in the a hove expression for $\Gamma_{\psi}$ vanishes. Using Eq. (9), we obtain $\Gamma_{\psi}$ in the form ready for easy applications:

$$
\Gamma_{\psi}=\left\langle\int_{P} d^{3} v \frac{I v_{\|}}{\Omega}\{Q\}\left(f_{0}\right)\right\rangle,
$$

where $\int_{P}$ represents that the velocity integril is to be evaluated only over the passing regime due to the fact that both $f_{0}$ and $\{Q\}$ are symmetric in $\eta_{\|}$in the trapped regime. We refer to Refs. 6 and 7 for the form of the bounce-averaged quasilinear ICRF-heating operator $\{Q\}$.

From this relation, we can easily see that we can have a nonzero $\Gamma_{\psi}$, if either $f_{0}$ or $\{Q\}$ is not symmetric in the sign of $v_{\|}$. The occurrence of a nonsymmetric $f_{0}$ is mo: $:$ obvious in the case of unbalanced neutral beam teating. The non-symmetric $v_{\|}$ behavior of $\{Q\}$ can be created by having a horizontal asymmetry in the RF-power density with unidirectional $k_{\|}$and using the Doppler shift effect to make particles with different $v_{\|\|}$resonate at different horizontal locations. A unidirectional toroidal wave spectrum can be generated using a series of phased radiating antenna elements. ${ }^{*}$

Since the absorbed RF power density $P_{\mathrm{RF}}$ can be expressed as

$$
P_{\mathrm{RF}}=\left\langle\int d^{3} v \in Q\left(f_{0}\right)\right\rangle=\int d^{3} v \in\{Q\}\left(f_{0}\right),
$$

where $\epsilon=m v^{2} / 2$ is the kinetic energy, we can estimate the magnitude of the above particle flux in terms of $P_{R F}$ as follows

$$
\Gamma_{\mathrm{v}} \approx \frac{P_{\mathrm{RF}}}{\epsilon_{\mathrm{0}}} \frac{I v_{0}}{\Omega}
$$

where $v_{0}$ is an average speed and $\epsilon_{0}=m v_{0}^{2} / 2$ is an average kinetic energy of the energetic ions. If we define an average minor radial distance $r$ for a noncircular flux surface using the toroidal flux $0=\bar{B}_{T \pi r^{2}}$, we have $\partial \psi / \partial r=\bar{R} \bar{B}_{P}$, where the 
overbars represent the flux-surface-averaged quantities. And thus we obtain

$$
\Gamma_{r}=\frac{\partial r}{\partial \psi \xi} \Gamma_{\psi}=\frac{1}{R B_{P}} \Gamma_{\psi} \sim \frac{P_{\mathrm{RF}}}{\epsilon_{0}} \rho_{p},
$$

where $\rho_{p}=v_{0} / \bar{\Omega}_{p}$ is the poloidal gyroradjus of energetic ions at the average velocity $r_{0}$

This is a very fast convective transport. For example, let us consider the case of fast ion control by use of spatially inhomogeneous RF wave power and apply it to a TFTR plasma with $5 \mathrm{MW}$ of controlling ICRF power absorbed by the neutralbeam-originated energetic hydrogen ions. If we localize the RF power to the outer half (larger major radius side) of the torus, but within the half minor radius, the $R F$ power will be confined in the volume of roughly $10 \mathrm{~m}^{3}$. This corresponds to about 0.5 uatt $/ \mathrm{cm}^{3}$ of absorbed RF power density. For the energetic hydrogen ions of density $1 \times 10^{13} \mathrm{~cm}^{-3}$ and average energy $50 \mathrm{keV}$, this much $\mathrm{RF}$ power can drive the radial convective transport speed as large as $50 \mathrm{~cm} / \mathrm{sec}$, using TFTR parameters at half minor radius and $q=2$. During one slowing-down time ( $\sim 1 s e c)$, the driven energetic ions can travel about half a minor raduis.

When we use nonuniform RF waves, the direction of the radial fux can te changed by changing the sign of $k_{\|}$. For the case where the RF-power is higher at larger major radius, positive $k_{\|}$yields positive radial transport. In the case where we have an asymmetric $f_{0}$ in $v_{\|}$, we do not need to have a directional $k_{\|}$. If the average $\bar{v}_{\|}$is positive, we have a radially outward flow, and vice versa. In the case where the background ions can also be resonant with the controlling waves, for an efficient coupling of the applied ICRF wave power to the high energy ions, higher harmonic heating is preferred, since some of the background ions at low energy could also be resonant and strongly coupled to the waves at the fundamental harmonic number.

The high rate of energetic ion transport with a moderate level of ICRF wave power predicted in the present theory opens up the possibility of centrolling energetic ion 
confinement in a tokamak. This scheme can be used to give inward pinch motion to the energetic ions generated by neutral beam or ICRF heating, and provide more efficient central heating of the plasma. By manipulating hot ion loss near the plasma edgs: we may also be able to create a large radial electric field in the edge region and study its effect on confinement (H-mode physics). It can also be used to the control of alpha ash accumulation by removing intermediate-energy alpha particles from the plasma as they slow down.

The author wishes to thank Dr. P. Colestock of PPPL for valuable discussions. This work was supported by the U.S. Department of Energy under Grant No. DE. FG02-86ER53223, and Contract No. DE-AC02-76-CH03073. 


\section{References}

${ }^{1}$ F.L. Hinto 7 and R.D. Hazeltine, Rev, Mod. Pl: 's. 48, 239 (1976).

A. Nocentini, M. Tessarotto, and F. Engelmann, Nucl. Fusion 15, 359(1975).

${ }^{3}$ C.S. Chang, G.W. Hammett, and R.J. Goldston, Princeton Plasma Physics Laboratory Report No. PPPL-2662(1989); submitted to Phys. Fluids.

${ }^{4}$ Peter J. Cato, Phys. Rev. Lett. 60, 1954(1988).

${ }^{5}$ Liu Chen, J. Vaclavik, and G. W. Hammett, Nucl. Fusion 28, 389(1988).

${ }^{6}$ I.B. Bernstein and D.C. Baxter, Phys. Fluids 24, 108(1981).

'T.H. Stix, Nucl Fusion 15, 737(1975).

${ }^{8}$ D.A. Ehst, in Proceedings of the 8th Topical Conference on Radic. Frequency Pawet in Plasmas (Irvine, CA, 1989) p. 393. 